\title{
Elastofibroma dorsi: Caso clínico y revisión de la literatura
}

\author{
David R. de Rungs-Brown, ${ }^{*} \bowtie$ José Manuel Mier-Odriozola, * Victor Baldin-Andre, \\ José Abel de la Peña-Salcedo,* Ricardo Balanzá-López*
}

*Hospital Ángeles Lomas; "Hospital General "Manuel Gea González". Trabajo recibido: 02-IX-2014; aceptado: 14-IV-2015

\begin{abstract}
RESUMEN. Los elastofibromas son un tipo de tumor raro benigno, de crecimiento lento originados a partir de tejidos blandos. El 99\% se localiza en región subescapular a causa de traumatismo continuo. Se presenta comúnmente con un tumor bien circunscrito, bien delimitado, no móvil, en la que no se adhiere a la piel suprayacente, sino a músculo y tejido subaponeurótico. El elastofibroma dorsi se manifiesta con dolor tipo punzante casi siempre moderado y progresivo al movimiento en abducción y aducción de la extremidad escapular afectada. Presentamos el siguiente estudio de un paciente masculino de 60 años de edad que fue diagnosticado histopatológicamente como un elastofibroma dorsi. El paciente presentaba una masa de tejido blando en la región dorsal y alteración funcional. En la exploración física se palpaba una masa adyacente subescapular; fue explorado con ecografía, radiografías, electrocardiograma, tomografía axial computarizada de tórax (TAC) y resonancia magnética $(\mathrm{RM})$. Se realiza un análisis del caso con el abordaje diagnóstico y terapéutico así como un reporte de la literatura. El elastofibroma dorsi es descrito como tumor del tejido conjuntivo, se pueden diagnosticar con mayor sensibilidad con la RM y menor especificidad con la TAC. En los tumores benignos la intervención quirúrgica es el método de elección; lo mismo sucede con el elastofibroma dorsi, presentando una mejoría clínica inmediata.
\end{abstract}

Palabras clave: Tumor benigno, tejido conectivo, tejido blando, región subescapular.

ABSTRACT. The elastofibromas are a rare type of slow-growing benign tumor arising from soft tissue. $99 \%$ of them occur in subscapular region due to continuous trauma. It usually presents with a well-defined, well-circumscribed tumor immobile in which it does not adhere to the overlying skin, if not subaponeurotic muscle and tissue. The elastofibroma dorsi manifests generally moderate and progressive movement in abduction and adduction of the shoulder gnawing pain affected limb. The study involved a male patient of 60 years old, who was diagnosed histopathologically as a dorsi elastofibroma. The patient showed a soft tissue mass in the dorsal region and functional impairment. On physical examination, an adjacent sub scapular mass was palpable. The patient was scanned with ultrasound, radiography, electrocardiography, computed tomography and magnetic resonance imaging. An analysis of the case with the diagnosis and treatment as well as a report of the literature was performed. The elastofibromas dorsi, is described as connective tissue tumor wich can be diagnosed with greater sensitivity with MRI and with lower specificity CT. In benign tumors surgery is the method of choice; is likewise in dorsi elastofibroma having immediate clinical improvement.

Key words: Benign tumor, connective tissue, soft tissue, subscapular region.

\section{INTRODUCCIÓN}

Los elastofibromas son un tipo de tumor benigno de crecimiento lento originados a partir de tejidos blandos. El 99\% se presenta en región subescapular, y ocasionalmente se pueden presentar de manera bilateral en el $2 \%$ de la población mayor a 50 años, la cual sugiere lesiones iguales o menores de $3 \mathrm{~cm}$ de diámetro. Las estadísticas obtenidas en este artículo demostraron una incidencia del $11.2 \%$ de hombres y $24.4 \%$ de mujeres. ${ }^{1}$

El elastofibroma dorsi fue descrito en 1961 por los médicos Järvi y Saxen, quienes lo describieron como un raro pseudotumor benigno del tejido blando conectivo fribroelástico no encapsulado de crecimiento lento. Normalmente se encuentra en el polo inferior de la escápula, en el fondo del serrato anterior y con frecuencia unida al periostio de las costillas. ${ }^{2}$

Objetivo: Presentar el caso de un paciente con elastofibroma dorsi izquierdo y describir el cuadro clínico junto con los métodos diagnósticos y terapéuticos de esta patología. Así como sistematizar la información en diferentes bases de datos del mismo.

\section{MATERIAL Y MÉTODOS}

El estudio incluyó a un paciente masculino de 60 años de edad, que fue diagnosticado histopatológicamente con un elastofibroma dorsi. El paciente presentaba una 
masa de tejido blando en la región dorsal y alteración funcional en miembro superior izquierdo.

En la exploración física se palpaba una masa adyacente subescapular. Se exploró y realizaron los siguientes métodos diagnósticos: ecografía, radiografías, electrocardiograma, tomografía axial computarizada de tórax (TAC) y resonancia magnética $(\mathrm{RM})$. Se realizó un análisis del caso con el abordaje diagnóstico y terapéutico, como también un reporte de la literatura.

Para el análisis de investigación fueron revisados artículos de los últimos 30 años (1982-2012). Posteriormente, de 20 artículos seleccionados éstos fueron probados para homogeneidad de datos mediante la razón de momios utilizando la prueba estadística BreslowDay. Se determinaron los signos y síntomas con mayor sensibilidad y especificidad del elastofibroma dorsi, así como su principal presentación, estudios diagnósticos y tratamiento con su pronóstico.

\section{CASO CLÍNICO}

Presentamos a un paciente masculino de 60 años de edad quien trabaja en un restaurante 14 horas diarias donde levanta objetos pesados, refiere tabaquismo positivo a razón de 30 cigarros al día desde los 15 años de edad y cuenta con antecedente de gota en 1998. No cuenta con más antecedentes de importancia.

Inició su padecimiento hace cinco años al presentar dolor moderado en región pectoral izquierda con irradiación a hemitórax izquierdo y región escapular izquierda, el cual se exacerbaba al realizar actividad física. El paciente no acudió a valoración médica debido a que la sintomatología se presentaba de manera intermitente; sin embargo, el cuadro de dolor se agudizó llegando a limitar los arcos de movilidad del hombro izquierdo, por lo que acudió a consulta médica. Para descartar patología cardiopulmonar fue sometido a protocolo de valoración a base de electrocardiograma, Holter, radiografía de tórax y espirometría, los cuales no arrojaron datos patológicos. Sus signos vitales estaban dentro de parámetros normales; a la exploración física se encontró una masa de consistencia dura, elástica, dolorosa a la palpación, de aproximadamente $5 \mathrm{~cm}$ de diámetro en región subescapular izquierda. Se realizó TAC donde puede observarse una tumoración ovoide con densidad semejante al músculo de la región en hemitórax izquierdo entre parrilla costal y vértice de escápula (figura 1). Debido a la sintomatología adversa que ocasionaba la tumoración hubo que dar manejo quirúrgico al paciente. Se le realizaron biometría hemática y coagulograma, la leucocitosis fue de 12,300 células $/ \mathrm{mcl}$, el resto de los parámetros de laboratorio estaban normales.

Bajo anestesia general y en decúbito lateral derecho se le practicó una incisión de aproximadamente 7 $\mathrm{cm}$ en la región subescapular izquierda. Se disecó el músculo dorsal ancho hasta llegar a una masa de tejido fibroelástico de aproximadamente $3 \times 5 \mathrm{~cm}$. La masa fue resecada, extraída y enviada para ser estudiada por el Servicio de Patología. Se realizó hemostasia del área quirúrgica y cerró en capas con Vicryl y Monocryl, después un vendaje compresivo en tórax.

El resultado final del Servicio de Patología fue un elastofibroma, no se observó neoplasia maligna. La descripción macroscópica reportó cortes seriados conformados de manera predominante por tejido adiposo, aunque con áreas blanquecinas, de consistencia elástica y aspecto de tejido fibroconectivo asociadas (figura 2). La descripción microscópica mostró degeneración eosinofílica parcial de la colágena superficial, células fusiformes estrelladas con componente fibroblástico y conectivo, datos de inflamación crónica granulomatosa con presencia de células gigantes multinucleadas y negatividad para neoplasia maligna (figura 2). No se realizaron estudios de inmunohistoquímica.
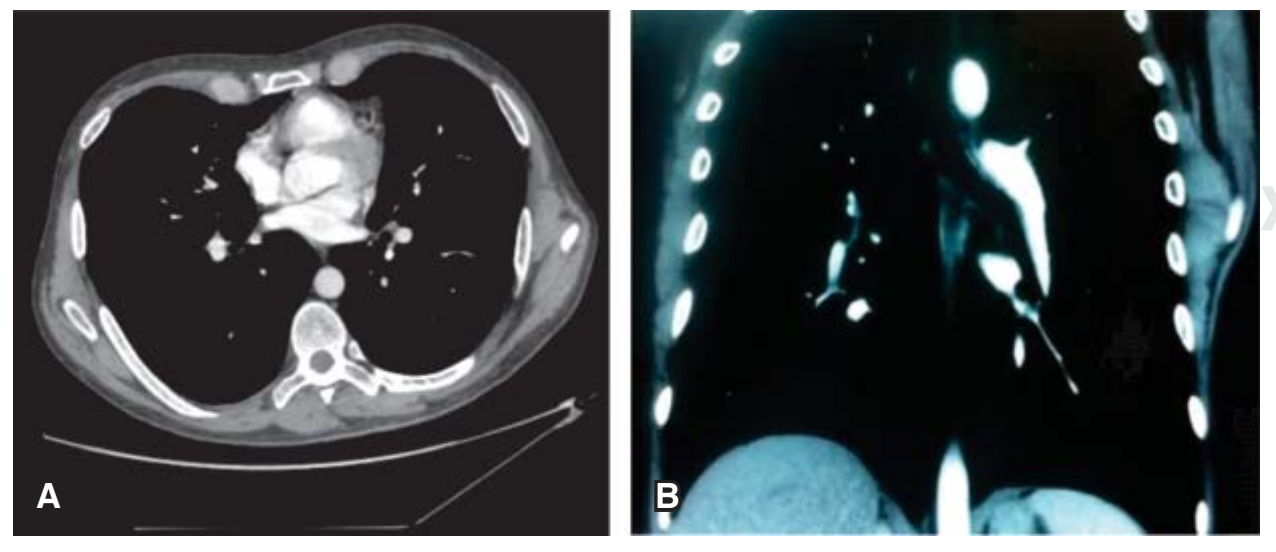

Figura 1.

Tomografía computada de tórax en la cual se observa tumoración ovoide con densidad semejante al músculo de la región en hemitórax izquierdo entre parrilla costal y el vértice de escápula. A) corte transversal, B) corte coronal. 


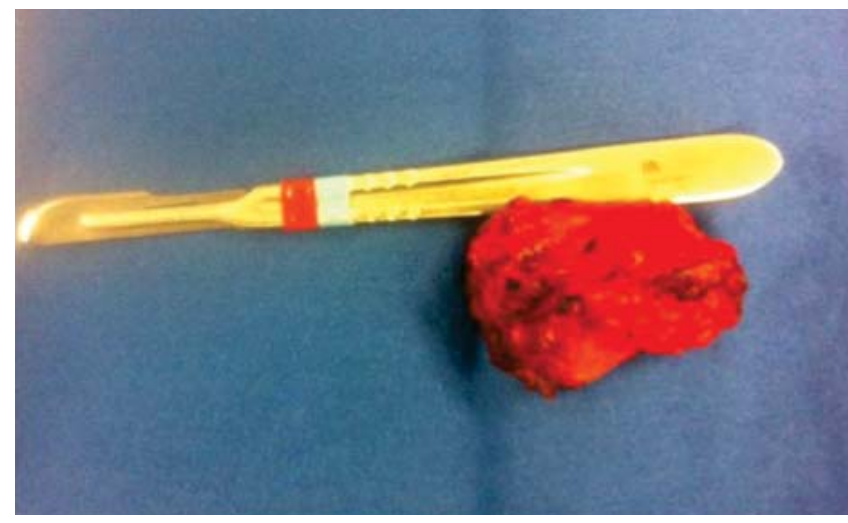

Figura 2. Tumoración en región subescapular izquierda de $5 \times 3 \mathrm{~cm}$ con componentes fibrosos y musculares. Bordes irregulares, mal definidas en una masa fibroelástica. La superficie de corte muestra líneas en blanco y amarillo del tejido causadas por el atrapamiento de restos grasos, similar a un «tablero». Tumores no encapsulados.

El paciente fue dado de alta dos días posteriores a la cirugía. No fue necesaria otra intervención quirúrgica o terapia adyuvante. Se presentó a consultas postoperatorias cada mes por cinco meses, encontrándose asintomático y sin datos de recurrencia.

\section{DISCUSIÓN}

El elastofibroma dorsi se presenta comúnmente como un tumor bien circunscrito, bien delimitado, no móvil, que no se adhiere a la piel suprayacente, sino a músculo y tejido subaponeurótico. La mayoría de los casos se encuentran en el área subescapular en un 98\%; también en los músculos gran dorsal en su parte anterior, músculo romboide en su porción superior y serratos anteriores en la porción dorsal. ${ }^{3}$ Existen otras áreas más infrecuentes como en el músculo deltoides. Asimismo, en porciones de roce continuo como en el trocánter mayor, olécranon y en la tuberosidad isquiática en un $2 \%$ de los casos. Esta manifestación clínica sólo se demuestra cuando el tumor invade tejidos circundantes fijándose al periostio subyacente. ${ }^{4}$

La fricción repetida en el área subescapular produciendo un trauma continuo, es un factor de riesgo e inductor del crecimiento de un producto anormal de la elastogénesis, produciendo una degeneración de fibras de colágena y activando el sistema de angiogénesis producido por la cascada de inflamación continua.

El elastofibroma dorsi puede presentarse de manera autosómica recesiva exponiendo el $33 \%$ de los pacientes con un familiar directo con este diagnóstico, generando la teoría de algún componente cromosómico desencadenante de la formación de éste. A pesar de que el tumor es unilateral puede presentarse en el $25 \%$ de los casos de forma bilateral. Además, se comporta como un tumor benigno siendo de crecimiento lento y puede llegar a tener un área de circunferencia de gran volumen, por lo general de $6 \times 6 \mathrm{~cm}$; sin embargo, un elastofibroma dorsi pequeño puede manifestarse con dolor al movimiento en abducción y aducción de la extremidad escapular afectada..$^{5,6}$

La radiografía, como estudio inicial, es considerada por la mayoría de los médicos como un método diagnóstico de descarte para alguna situación ortopédica que involucre la pared o parrilla torácica presentándose en su mayoría normal, al menos que haya deformación de la anatomía en la región estudiada por el tamaño del tumor; detectando elevación importante de la escápula o el tejido óseo adyacente. La TAC y RM revelan una masa bien circunscrita de los tejidos blandos. Los elastofibromas dorsi descritos como tumores del tejido conjuntivo se pueden visualizar con mayor sensibilidad con la RM y menor especificad con la TAC, ya que pueden tener un pedículo nutricio vascular; el medio de contraste puede determinar con mayor sensibilidad el área de presencia tumoral, sobre todo con el uso del gadolinium. ${ }^{7,8}$

Como todo tumor encontrado, debe realizarse un estudio de histología por patología, obteniendo una biopsia excisional para un estudio transoperatorio o definitivo en el área afectada. El tejido enviado tiene que incluir tejido adyacente para delimitar áreas afectadas y delimitar bordes. También debe incluir piel, tejido subcutáneo y tejido blando profundo y, en ocasiones, limítrofes de tejido óseo. ${ }^{9}$

El elastofibroma dorsi macroscópicamente se comporta como un tumor bien definido de superficie irregular con coloración gris-blanca con componente fibroso y componente vascular. Casi siempre con un tamaño de 6 a $10 \mathrm{~cm}$ de diámetro.

En la descripción microscópica de los elastofibromas se notan células fusiformes estrelladas con componente fibroblasto y conectivo por tinción de Russell-Movat, siendo positivas por la inmunohistoquímica con vimentina para determinar músculo. Se describen paquetes eosinófilos de colágeno en la periferia.10,11 Se encontró componente elasticofibroso con las tinciones de ReyesMota (figura 3). ${ }^{12}$

En los tumores benignos la intervención quirúrgica es el tratamiento de elección; de igual modo, en el elastofibroma dorsi el mejor tratamiento es la resección total de la tumoración. Por lo regular, el tumor puede llegar a ser progresivo, incrementando los síntomas de acuerdo al tamaño. Una vez diagnosticado el tumor, hay que realizar una exegesis tumoral, teniendo como resultados una mejoría total 

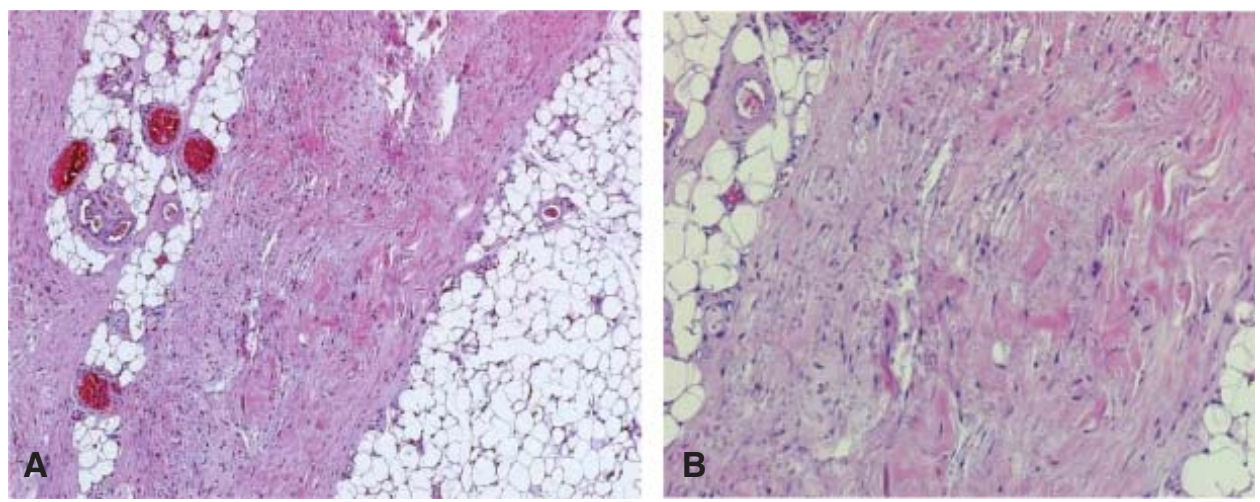

Figura 3. Estudio histopatológico de elastofibroma dorsi. A) tejido elástico con estructuras formando glóbulos o discos. En la tinción de Van Gieson las fibras son teñidas de color marrón oscuro a negro. Las lesiones son predominantemente hipocelulares con tumoración fibroblástica y células sin atipia y actividad mitótica, B) tumor compuesto de tejido fibroso, colagenoso y alargado. Ronda en forma de fibras elásticas densas.

e inmediata de los síntomas. No existe tratamiento conservador del mismo. ${ }^{13,14}$

Los principales diagnósticos diferenciales del elastofibroma dorsi son el pseudotumor inflamatorio y el fibrohistiocitoma maligno, debido a que presentan un cuadro clínico similar. El pseudotumor inflamatorio es un término utilizado para describir un proceso benigno y poco común que afecta mayormente a pulmón y órbita. Este tumor se presenta como una masa de células inflamatorias polimórficas acompañadas de diversos grados de fibrosis, necrosis, reacción granulomatosa y células miofibroblásticas. En estudios radiográficos esta entidad se observa como una lesión única, con bordes bien definidos, lobulada, de predominio en lóbulos inferiores. El tratamiento recomendado del pseudotumor inflamatorio es la resección radical del tumor, en casos en los cuales no se pueda realizar un intervención quirúrgica se recomienda radioterapia. ${ }^{15} \mathrm{El}$ fibrohistiocitoma maligno es el sarcoma de partes blandas más común en la población adulta. Esta neoplasia puede encontrase en cualquier órgano que derive de tejido mesenquimatoso. Histológicamente está conformado por células fusiformes, histiocitarias, gigantes y zonas de pleomorfismo. Su inmunohistoquímica usualmente es positiva para vimentina, actina, CD-68 y alfa1-antitripsina. El tratamiento de elección en estos casos es la resección completa con márgenes libres $y$ en casos irresecables la radioterapia. ${ }^{16,17}$

\section{CONCLUSIÓN}

A pesar que el elastofibroma dorsi es poco común, se ha estudiado que hay una coexistencia con un sarcoma fusocelular de alto grado y un leiomiosarcoma de alto grado. Por otra parte, también es asociado a antecedentes de lipomas, metástasis, sarcomas, fibromatosis extra abdominal y hemangiomas en la parte posterior y en la región subescapular. Se puede relacionar a pacientes con antecedentes de múltiple frecuencia de fricción en región subescapular. El diagnóstico se confirma con la TAC y biopsia. Buen pronóstico.

\section{REFERENCIAS}

1. Hidalgo Grau LA, Ardevol J, Soler T, Auleda J, Ubach M. Elastofibroma dorsi: clinical and pathologic aspects of two cases. Acta Orthop Belg 1995;61(4):302-304.

2. Järvi $\mathrm{OH}$, Saxen E. Elastofibroma dorsi. Acta Pathol Microbiol Scand Suppl 1961;51(suppl 144):83-84.

3. Rosell PJ, Ramírez TC, Carazo MO, Guerrero FJA, Vara TR. Elastofibroma dorsi. Rev Esp Cir Osteoart 1992;27(159):171-174.

4. Järvi $\mathrm{OH}$, Länsimies PH. Subclinical elastofibromas in the scapular region in an autopsy series. Acta Pathol Microbiol Scand A 1975;83(1):87-108.

5. Hoven-Gondrie ML, IJpma FF, Havenith MG, van Geldere D. Elastofibroma dorsi: a characteristic, benign, subscapular swelling. Ned Tijdschr Geneeskd 2009;153:A569.

6. Baudrez V, Malghem J, Van de Berg B, Lebon C, Lecouvet $\mathrm{F}$, Maldague B. Aspect échographique de l'élastofibrome dorsal: A propos de 6 cas. J Radiol 1998;79(6):549-551.

7. Berthoty DP, Shulman HS, Miller HA. Elastofibroma: chest wall pseudotumor. Radiology 1986;160(2):341342.

8. Brandser EA, Goree JC, El-Khoury GY. Elastofibroma dorsi: prevalence in an elderly patient population as revealed by CT. AJR Am J Roentgenol 1998;171(4):977980.

9. Charissoux JL, Mabit C, Fiorenza F, Gougam T, Leboutet MJ. Elastofibroma in the scapular region. A case report 
and review of the literature. Rev Chir Orthop Reparatrice Appar Mot 2000;86(1):98-103.

10. Nishida A, Uetani M, Okimoto T, Hayashi K, Hirano T. Bilateral elastofibroma of the thighs with concomitant subscapular lesions. Skeletal Radiol 2003;32(2):116-118.

11. Pyne D, Mootoo R, Bhanji A, Amin S. Elastofibroma dorsi [Letters]. Ann Rheum Dis 2002;61(3):278-279.

12. Soler R, Requejo I, Pombo F, Sáez A. Elastofibroma dorsi: MR and CT findings. Eur J Radiol 1998;27(3):264-267.

13. Turna A, Yilmaz MA, Urer N, Bedirhan MA, Gürses A. Bilateral elastofibroma dorsi. Ann Thorac Surg 2002;73(2):630-632.

14. Vanni R, Marras S, Faa G, et al. Chromosome instability in elastofibroma. Cancer Genet Cytogenet 1999;111(2):182-183.

15. Yu JS, Weis LD, Vaughan LM, Resnick D. MRI of elastofibroma dorsi. J Comput Assist Tomogr 1995;19(4):601-603.
16. Patnana $M$, Sevrukov $A B$, Elsayes $K M$, Viswanathan $C$, Lubner M, Menias CO. Inflammatory pseudotumor: the great mimicker. AJR Am J Roentgenol 2012;198(3):W217227. doi: 10.2214/AJR.11.7288.

17. Oda Y, Tamiya S, Oshiro Y, et al. Reassesment and clinicopathological prognostic factors of malignant fibrous histiocytoma of soft parts. Pathol Int 2002;52(9):595606.

\section{$\triangle$ Correspondencia:}

Dr. David R. de Rungs Brown

Hospital Ángeles Lomas.

Correo electrónico: De_rungs@hotmail.com

Los autores declaran no tener conflicto de intereses. 